\title{
Large-scale velocities and primordial non-Gaussianity
}

\author{
Fabian Schmidt \\ Theoretical Astrophysics, California Institute of Technology M/C 350-17, Pasadena, California 91125, USA
}

(Received 1 June 2010; published 1 September 2010)

\begin{abstract}
We study the peculiar velocities of density peaks in the presence of primordial non-Gaussianity. Rare, high-density peaks in the initial density field can be identified with tracers such as galaxies and clusters in the evolved matter distribution. The distribution of relative velocities of peaks is derived in the large-scale limit using two different approaches based on a local biasing scheme. Both approaches agree, and show that halos still stream with the dark matter locally as well as statistically, i.e. they do not acquire a velocity bias. Nonetheless, even a moderate degree of (not necessarily local) non-Gaussianity induces a significant skewness $(\sim 0.1-0.2)$ in the relative velocity distribution, making it a potentially interesting probe of nonGaussianity on intermediate to large scales. We also study two-point correlations in redshift space. The well-known Kaiser formula is still a good approximation on large scales, if the Gaussian halo bias is replaced with its (scale-dependent) non-Gaussian generalization. However, there are additional terms not encompassed by this simple formula which become relevant on smaller scales $(k \gtrsim 0.01 \mathrm{~h} / \mathrm{Mpc})$. Depending on the allowed level of non-Gaussianity, these could be of relevance for future large spectroscopic surveys.
\end{abstract}

DOI: 10.1103/PhysRevD.82.063001

PACS numbers: 95.30.Sf, 95.36.+x, 98.80.-k, 98.80.Jk

\section{INTRODUCTION}

Observations of the large-scale structure in the Universe that use galaxies, clusters, or other tracers of the density field are done in redshift space: the distance is generally inferred using the redshift $z$, which receives a contribution from the line-of-sight velocity of the object. These velocities are due to the gravitational field which is correlated with the density field itself. On large scales where linear perturbation theory in the density field applies, the leading contribution is the squashing (or stretching, in case of an underdensity) of a volume element in redshift space relative to real space. In this limit, there is a simple relation between the real- and redshift-space power spectra, $P_{g}$ and $P_{g, s}$, respectively, of a tracer " $g$ " $[1,2]$ :

$$
P_{g, s}(k, \mu)=\left(1+\frac{f}{b_{1}} \mu^{2}\right)^{2} P_{g}(k),
$$

where $\mu$ is the cosine of the $\mathbf{k}$ vector with the line of sight, $f=d \ln D / d \ln a$ is the logarithmic derivative of the linear growth factor, and $b_{1}$ is the linear bias of the tracer population. Apart from the large-scale, small-correlation limit, the relation Eq. (1) makes two assumptions: first, that the tracer population is characterized by a deterministic, local, and, on large scales, linear bias. In particular, if the tracer density $\delta_{g}(\mathbf{x})$ is a local function $F(\delta(\mathbf{x}))$ of the matter density perturbation $\delta$, we can expand in $\delta(\mathbf{x})$ to obtain [3]

$$
\delta_{g}(\mathbf{x})=b_{1} \delta(\mathbf{x})+\frac{b_{2}}{2} \delta^{2}(\mathbf{x})+\ldots,
$$

where the bias parameters are either to be seen as free empirical parameters, or can be determined using various theoretical approaches. Local biasing is also derivable from the conjecture that dark matter halos form in highdensity regions (peaks) in the initial density field. This assumption holds well in the high-peak/massive halo regime, which we assume throughout. Hence, in the following we will somewhat loosely use "peaks" and "halos" interchangeably.

The second assumption used for Eq. (1) is that the cosmological density field is Gaussian on large scales. While we will retain the local biasing scheme, we are interested in relaxing the second assumption of Gaussianity. Recently, there has been renewed interest in probing the Gaussianity of the primordial seed perturbations via large-scale structure (see [4] for a review). The simplest way of obtaining a non-Gaussian field is by adding a local nonlinearity:

$$
\Phi(\mathbf{x})=\Phi_{\mathrm{G}}(\mathbf{x})+f_{\mathrm{NL}}\left(\Phi_{\mathrm{G}}^{2}(\mathbf{x})-\left\langle\Phi_{\mathrm{G}}^{2}\right\rangle\right),
$$

where $\Phi_{\mathrm{G}}$ is a Gaussian random field, and $\Phi$ is the resulting non-Gaussian field. Of course, one can add higher powers to the series Eq. (3), though the quadratic term usually has the largest impact. Following standard convention, we let $\Phi$ stand for the primordial potential, related to the density field through the transfer function and Poisson equation (see Appendix A).

As shown by [5] and confirmed by [6,7], in the presence of non-Gaussian initial conditions of the local type, halos acquire a scale-dependent correction to their bias which becomes important on large scales:

$$
b_{1} \rightarrow b_{1}+2 f_{\mathrm{NL}}\left(b_{1}-1\right) \delta_{c} \mathcal{M}(k)
$$


where $\mathcal{M}(k) \propto k^{-2}$ is the relation between density and potential in Fourier space (see Appendix A).

Besides the local model, several other possible bispectrum configurations have been proposed in the literature, such as the equilateral (e.g., [8]) and folded types (e.g., [9]). We summarize these types of non-Gaussianity and the relation between the potential and matter perturbations in Appendix A.

Given the significant impact of (local) non-Gaussianity on the power spectrum of biased tracers, it is then natural to ask what happens to the power spectrum in redshift space, and whether Eq. (1) still holds. Furthermore, the distribution of relative velocities between tracers at $\mathbf{x}_{1}$ and $\mathbf{x}_{2}$,

$$
\delta \mathbf{u}=\mathbf{u}\left(\mathbf{x}_{2}\right)-\mathbf{u}\left(\mathbf{x}_{1}\right)
$$

is itself an interesting probe of non-Gaussianity [10].

In this paper, we assume subhorizon scales throughout, and adopt the Newtonian gauge; further, we work in Lagrangian coordinates. At first order, the transition to Eulerian coordinates (which all observations as well as simulation measurements are made in) simply amounts to replacing the Lagrangian bias parameters with their Eulerian counterparts, in the case of the linear bias simply $b_{E, 1}=b_{L, 1}+1$.

While we will focus on the local type of primordial nonGaussianity for the most part, the expressions obtained can easily be evaluated for any given primordial bispectrum, and we will show selected results for other bispectrum shapes.

The paper is structured as follows: Section II introduces density and velocity correlations, and some notation. Section III contains two different derivations of the moments of the relative peak velocity distribution. We discuss the distribution of peak velocities in Sec. IV. Finally, Sec. V presents the power spectrum of peaks (halos) in redshift space. We conclude in Sec. VI.

\section{PRELIMINARIES: DENSITY AND VELOCITY FIELDS}

We consider the linear overdensity and velocity fields of matter $\delta(\mathbf{x}), \mathbf{v}(\mathbf{x})$ as random fields, related by the (linear) continuity equation,

$$
\dot{\delta}+\nabla \mathbf{v}=0 .
$$

Since for the linear density field $\dot{\delta}=a H f \delta$, we can define a scaled velocity $\mathbf{u}$ which satisfies

$$
\mathbf{u} \equiv \frac{\mathbf{v}}{a H f} \Rightarrow \nabla \mathbf{u}=-\delta .
$$

In the following, we will always deal with smoothed density and velocity fields which are indicated by a subscript $R$ :

$$
\delta_{R}(\mathbf{x})=\int \mathrm{d}^{3} \mathbf{y} W_{R}(|\mathbf{x}-\mathbf{y}|) \delta(\mathbf{x}),
$$

$$
\mathbf{u}_{R}(\mathbf{x})=\int \mathrm{d}^{3} \mathbf{y} W_{R}(|\mathbf{x}-\mathbf{y}|) \mathbf{u}(\mathbf{x}),
$$

where $W_{R}$ is a normalized window function. Specifically, we use a real-space tophat filter for $W_{R}$, though the shape of the window function has negligible impact on our results. Following the standard convention, we choose the smoothing scale $R$ corresponding to a halo of mass $M$ to be determined by $R=(3 M / 4 \pi \bar{\rho})^{1 / 3}$, where $\bar{\rho}$ is the background matter density.

Below, we will need two-point correlations of the density, velocity, and the cross correlation between the two. Using the smoothed matter power spectrum,

$$
P_{R}(k)=\tilde{W}_{R}^{2}(k) P(k)
$$

where $P(k)$ is the unsmoothed power spectrum and $\tilde{W}$ is the Fourier transform of the window function, the two-point correlations are given by

$$
\begin{gathered}
\xi_{R}=\xi_{R}^{\delta \delta}(r) \equiv\left\langle\delta_{R}\left(\mathbf{x}_{1}\right) \delta_{R}\left(\mathbf{x}_{2}\right)\right\rangle \\
=\frac{1}{2 \pi^{2}} \int \mathrm{d} k k^{2} P_{R}(k) j_{0}(k r), \\
\xi_{R}^{u \delta}(r) \equiv\left\langle\delta_{R}\left(\mathbf{x}_{1}\right) \hat{\mathbf{r}} \cdot \mathbf{u}_{R}\left(\mathbf{x}_{2}\right)\right\rangle=-\frac{1}{2 \pi^{2}} \int \mathrm{d} k k P_{R}(k) j_{1}(k r), \\
\xi_{R}^{u u}(r) \equiv \frac{1}{3}\left\langle\mathbf{u}_{R}\left(\mathbf{x}_{1}\right) \cdot \mathbf{u}_{R}\left(\mathbf{x}_{2}\right)\right\rangle=\frac{1}{6 \pi^{2}} \int \mathrm{d} k P_{R}(k) j_{0}(k r) .
\end{gathered}
$$

Here, $r=\left|\mathbf{x}_{2}-\mathbf{x}_{1}\right|$ and $\hat{\mathbf{r}}=\left(\mathbf{x}_{2}-\mathbf{x}_{1}\right) / r$, and the $j_{n}$ denote spherical Bessel functions. $\xi_{R}^{u \delta}(r)$ is the crosscorrelation function between the density at point $\mathbf{x}_{1}$ and the matter velocity at point $\mathbf{r}_{2}$ projected along the separation vector. It is negative, since in the presence of an overdensity $\delta_{R}\left(\mathbf{x}_{1}\right)>0$ the streaming motion will be directed towards point $\mathbf{x}_{1}$. Finally, we define the variance of the smoothed density field $\sigma_{R}$ and the one-dimensional smoothed velocity dispersion $\sigma_{u}$ :

$$
\sigma_{R}^{2} \equiv \xi_{R}^{\delta \delta}(0) ; \quad \sigma_{u}^{2} \equiv \xi_{R}^{u u}(0) .
$$

We will also need expressions for various three-point correlators of the velocity (projected on r) and density. The density three-point function is given in terms of the smoothed matter bispectrum $B_{R}$, which is defined in Appendix A, by

$$
\begin{aligned}
\xi_{R}^{\delta \delta \delta}\left(\mathbf{x}_{1}, \mathbf{x}_{2}, \mathbf{x}_{3}\right)= & \int \frac{\mathrm{d}^{3} \mathbf{k}_{1}}{(2 \pi)^{3}} \int \frac{\mathrm{d}^{3} \mathbf{k}_{2}}{(2 \pi)^{3}} e^{i\left[\mathbf{k}_{1} \cdot\left(\mathbf{x}_{1}-\mathbf{x}_{3}\right)+\mathbf{k}_{2} \cdot\left(\mathbf{x}_{2}-\mathbf{x}_{3}\right)\right]} \\
& \times B_{R}\left(\mathbf{k}_{1}, \mathbf{k}_{2},-\mathbf{k}_{1}-\mathbf{k}_{2}\right) .
\end{aligned}
$$

Since we are interested in two-point peak correlations, we will always encounter degenerate triangles with $\mathbf{x}_{3}=\mathbf{x}_{2}$. If instead two other vertices coincide, we can always relabel the indices to bring the correlation into this form. 
We define the general, mixed density/velocity degenerate three-point correlation as

$$
\begin{gathered}
\xi_{R}^{l m n}(r)=\left\langle X^{(l)}\left(\mathbf{x}_{1}\right) X^{(m)}\left(\mathbf{x}_{1}\right) X^{(n)}\left(\mathbf{x}_{2}\right)\right\rangle, \\
X^{(l)}(\mathbf{x})= \begin{cases}\delta_{R}(\mathbf{x}), & l=0 \\
\hat{\boldsymbol{r}} \cdot \mathbf{u}_{R}(\mathbf{x}), & l=1,\end{cases}
\end{gathered}
$$

where $\mathbf{r}=\mathbf{x}_{2}-\mathbf{x}_{1}$. Using that in Fourier space, $\tilde{\mathbf{u}}_{R} \cdot \hat{\boldsymbol{r}}=$ $\hat{\boldsymbol{k}} \cdot \hat{\boldsymbol{r}} /(-i k) \tilde{\delta}_{R}$, and noting our definition of $\mathbf{r}$, we can write these correlations as

$$
\begin{aligned}
\xi_{R}^{l m n}(r)= & \int \frac{\mathrm{d}^{3} \mathbf{k}}{(2 \pi)^{3}} e^{i \mathbf{k} \cdot \mathbf{r}}\left(\frac{\hat{\boldsymbol{k}} \cdot \hat{\boldsymbol{r}}}{-i k}\right)^{n} \int \frac{\mathrm{d}^{3} \mathbf{k}_{2}}{(2 \pi)^{3}}\left(\frac{\hat{\boldsymbol{k}}_{2} \cdot \hat{\boldsymbol{r}}}{-i k_{2}}\right)^{m} \\
& \times\left(\frac{-\left(\mathbf{k}+\mathbf{k}_{2}\right) \cdot \hat{\boldsymbol{r}}}{-i\left|\mathbf{k}+\mathbf{k}_{2}\right|^{2}}\right)^{l} B_{R}\left(\mathbf{k}+\mathbf{k}_{2}, \mathbf{k}_{2}, \mathbf{k}\right) .
\end{aligned}
$$

The corresponding Fourier-space quantity can be defined by

$$
\begin{aligned}
\xi_{R}^{l m n}(r)= & \int \frac{\mathrm{d}^{3} \mathbf{k}}{(2 \pi)^{3}} e^{i \mathbf{k} \cdot \mathbf{r}} i^{l+m+n} \mathcal{P}^{l m n}(\mathbf{k}), \\
\mathcal{P}^{l m n}(k)= & \left(\frac{1}{k}\right)^{n} \int \frac{\mathrm{d}^{3} \mathbf{k}_{2}}{(2 \pi)^{3}}\left(\frac{-k_{z}-k_{2 z}}{\left|\mathbf{k}+\mathbf{k}_{2}\right|^{2}}\right)^{l}\left(\frac{k_{2 z}}{k_{2}^{2}}\right)^{m} \\
& \times B_{R}\left(\mathbf{k}+\mathbf{k}_{2}, \mathbf{k}_{2}, \mathbf{k}\right),
\end{aligned}
$$

where here and throughout we use the small-angle (flat sky) approximation, and let the $\mathbf{r}$ vector be along the $z$ axis. Further, we have taken out powers of $i$ in order to make $\mathcal{P}^{l m n}$ real.

For the numerical results, we adopt a flat $\Lambda \mathrm{CDM}$ cosmology, with $\Omega_{m}=1-\Omega_{\Lambda}=0.28, \Omega_{b}=0.046, \sigma_{8}=$ 0.8 , and $n_{s}=0.958$. The Gaussian Lagrangian bias of halos is calculated as $b_{L}=\delta_{c} / \sigma_{R}^{2}$, where $\delta_{c}=1.686$ is the linear collapse threshold. Note that our expressions are more general in the sense that they can be used with any local bias parameters (see Sec. III A).

\section{VELOCITIES OF DENSITY PEAKS}

In this section we derive the moments of the distribution of velocities of density peaks in two different ways, both based on the local bias model of Eq. (2) (i.e., local in terms of the non-Gaussian density field $\delta$ ). We are interested in the relative velocity of two peaks projected on the separation vector,

$$
\delta u \equiv\left[\mathbf{u}\left(\mathbf{x}_{2}\right)-\mathbf{u}\left(\mathbf{x}_{1}\right)\right] \cdot \hat{\mathbf{r}} .
$$

This is the relevant quantity to compare with observations of large-scale velocities, in particular, redshift-space distortions (see Sec. V). Homogeneity and isotropy dictate that the statistical properties of $\delta u$ such as its moments only depend on $r=\left|\mathbf{x}_{2}-\mathbf{x}_{1}\right|$.

In order to obtain the moments of the peak velocity distribution, we have to weight each location in the volume average by its peak density. In other words, the average relative velocity of peaks is given by

$$
\begin{aligned}
\langle\delta u\rangle_{\mathrm{pk}} & =\frac{\left\langle n_{\mathrm{pk}}\left(\mathbf{x}_{1}\right) n_{\mathrm{pk}}\left(\mathbf{x}_{2}\right) \delta u\right\rangle}{\left\langle n_{\mathrm{pk}}\left(\mathbf{x}_{1}\right) n_{\mathrm{pk}}\left(\mathbf{x}_{2}\right)\right\rangle} \\
& =\frac{1}{1+\xi_{\mathrm{pk}}(r)}\left\langle\left( 1+\delta_{\mathrm{pk}}\left(\mathbf{x}_{1}\right)\left(1+\delta_{\mathrm{pk}}\left(\mathbf{x}_{2}\right) \delta u\right\rangle,\right.\right.
\end{aligned}
$$

and similarly for higher moments of the relative velocity. Here, $\xi_{\mathrm{pk}}$ is the correlation function of peaks. Since we are only dealing with the lowest order in perturbation theory and $\delta u$ is already first order, we will neglect the normalizing prefactor in the following.

\section{A. Unbiased velocity ansatz}

The first, much simpler approach is to assume that the velocities of peaks are locally and statistically unbiased. The absence of a local velocity bias is motivated by the fact that local processes such as gravitational collapse of smallscale overdensities should not induce any relative velocity with respect to the large-scale flow of matter. In Gaussian $N$-body simulations, this ansatz has been shown to hold well on large scales, and one would not expect that mildly non-Gaussian initial conditions will change this.

On the other hand, as shown in $[11,12]$, density peaks have a statistical, scale-dependent velocity bias when the peak constraint is employed. Here, statistical means that each halo still moves with the dark matter locally (no local velocity bias), but a velocity bias still comes about from the fact that peaks in the density field are in special locations. This velocity bias from the peak constraint scales as $k^{2}$, and we will neglect it here for simplicity since we are interested in large scales. However, it is not apparent whether a nonGaussianity of the density field on large scales can also source such a statistical velocity bias. We defer this question to Sec. III B, and assume in this section that there is no such bias.

Using the ansatz of unbiased velocities, it is easily possible to derive the first few moments of the velocity difference distribution. For this, we expand the peak density to second order (in Lagrangian space):

$$
\delta_{\mathrm{pk}}(\mathbf{x})=b_{L} \delta_{R}(\mathbf{x})+\frac{1}{2} b_{L, 2} \delta_{R}^{2}(\mathbf{x}),
$$

where the second order bias parameter is $b_{L, 2}=b_{L}^{2}$ in the high-peak (high-significance) limit we work in (see below for the general result). For the sake of clarity, we will omit the subscript $R$ for the smoothed fields, and let $\delta_{i}=\delta_{R}\left(\mathbf{x}_{i}\right)$ and similarly for $u$ for the remainder of this section.

By assumption, $\mathbf{u}_{\mathrm{pk}}=\mathbf{u}$, and we have for the relative velocity (Eq. (21), to first order in the correlations): 


$$
\begin{aligned}
\langle\delta u\rangle_{\mathrm{pk}}(r)= & \left\langle\left[1+b_{L} \delta_{1}+\frac{1}{2} b_{L, 2} \delta_{1}^{2}\right]\left[1+b_{L} \delta_{2}+\frac{1}{2} b_{L, 2} \delta_{2}^{2}\right] \hat{\mathbf{r}}\right. \\
& \left.\cdot\left[\mathbf{u}_{2}-\mathbf{u}_{1}\right]\right\rangle \\
= & 2 b_{L} \xi_{R}^{u \delta}(r)+2 b_{L}^{2} \xi_{R}^{\delta \delta u}\left(\mathbf{x}_{1}, \mathbf{x}_{2}, \mathbf{x}_{2}\right) \\
& +b_{L, 2} \xi_{R}^{\delta \delta u}\left(\mathbf{x}_{1}, \mathbf{x}_{1}, \mathbf{x}_{2}\right) \\
= & 2 b_{L} \xi_{R}^{u \delta}(r)-2 b_{L}^{2} \xi_{R}^{\delta u \delta}(r)+b_{L}^{2} \xi_{R}^{\delta \delta u}(r) .
\end{aligned}
$$

Here we have used Eq. (16) to express the various threepoint correlations in a uniform way. Note that when reordering the arguments of the three-point function in order to bring them into the form of Eq. (16), this can entail a change of sign when swapping $\mathbf{x}_{1} \leftrightarrow \mathbf{x}_{2}$ for terms odd in $u$ (due to the projection onto $\hat{\mathbf{r}}$ ).

We see that while we recover the standard expression in the Gaussian case (obtained by setting all three-point functions to zero; see e.g., [11]), there are several threepoint terms contributing to the mean relative velocity. Note however that $\langle\delta u\rangle_{\mathrm{pk}}$ is nonzero only for biased tracers $b_{L} \neq 0$ even in the presence of non-Gaussianity.

Next, the dispersion of velocity differences is given by

$$
\begin{aligned}
\left\langle\delta u^{2}\right\rangle_{\mathrm{pk}}(r)= & \left\langle\left[1+b_{L} \delta_{1}\right]\left[1+b_{L} \delta_{2}\right]\left(\hat{\mathbf{r}} \cdot\left[\mathbf{u}_{2}-\mathbf{u}_{1}\right]\right)^{2}\right\rangle \\
= & 2 \sigma_{u}^{2}+2 \xi_{R}^{u u}(r)+2 b_{L}\left\langle\delta_{1}\left(\hat{\mathbf{r}} \cdot\left[\mathbf{u}_{2}-\mathbf{u}_{1}\right]\right)^{2}\right\rangle \\
= & 2 \sigma_{u}^{2}+2 \xi_{R}^{u u}(r) \\
& +2 b_{L}\left[\xi_{R}^{u u} \delta(0)+\xi_{R}^{u u} \delta(r)-2 \xi_{R}^{\delta u u}(r)\right] .
\end{aligned}
$$

Again, we recover the standard expression in the Gaussian case, with several additional three-point contributions. These contributions vanish however for an unbiased tracer $\left(b_{L}=0\right)$.

It is straightforward to generalize $\langle\delta u\rangle_{\mathrm{pk}}$ and $\left\langle\delta u^{2}\right\rangle_{\mathrm{pk}}$ to the case when two different tracers with arbitrary linear and quadratic bias parameters are considered. The first two moments of the relative velocity distribution between different tracers are then given by

$$
\begin{aligned}
\langle\delta u\rangle_{\mathrm{pk}}(r)= & \left(b_{1 a}+b_{1 b}\right) \xi_{R}^{u \delta}(r)-2 b_{1 a} b_{1 b} \xi_{R}^{\delta u \delta}(r) \\
+ & \frac{1}{2}\left(b_{2 a}+b_{2 b}\right) \xi_{R}^{\delta \delta u}(r) . \\
\left\langle\delta u^{2}\right\rangle_{\mathrm{pk}}(r)= & 2 \sigma_{u}^{2}+2 \xi_{R}^{u u}(r)+\left(b_{1 a}+b_{1 b}\right) \\
& \times\left[\xi_{R}^{u u \delta}(0)+\xi_{R}^{u u \delta}(r)-2 \xi_{R}^{\delta u u}(r)\right] .
\end{aligned}
$$

Here, $b_{1 a}, b_{1 b}$ denote the linear (Lagrangian or Eulerian) bias parameters for the two tracers, while $b_{2 a}, b_{2 b}$ denote the corresponding quadratic biases [Eq. (2)].

Finally, there is also a third moment of $\delta u$, resulting in a skewed velocity difference distribution as already noticed by [10]. It is given by

$$
\begin{aligned}
\left\langle\delta u^{3}\right\rangle_{\mathrm{pk}}(r) & =\left\langle\left[1+b_{L} \delta_{1}\right]\left[1+b_{L} \delta_{2}\right]\left(\hat{\mathbf{r}} \cdot\left[\mathbf{u}_{2}-\mathbf{u}_{1}\right]\right)^{3}\right\rangle \\
& =\left\langle\left[\hat{\mathbf{r}} \cdot\left(\mathbf{u}_{2}-\mathbf{u}_{1}\right)\right]^{3}\right\rangle=6 \xi_{R}^{\text {uиu }}(r),
\end{aligned}
$$

where for the second equality we have again assumed the absence of any four- and higher-point terms. The third moment of velocities is obviously absent in the Gaussian case, but for non-Gaussian initial conditions it is nonzero even for unbiased tracers (e.g., matter itself). In the next section we outline the second derivation of these results (more details can be found in Appendix B).

\section{B. Derivation in statistical field theory}

The assumption that peak velocities are still statistically unbiased in the presence of large-scale non-Gaussianity seems natural. On the other hand, identifying peaks in the density field is a nonlinear process, and thus it is desirable to have a proof for this assumption. Our second derivation of the distribution of relative velocities provides such a proof without making any assumptions apart from the large-scale limit (i.e., small correlations), and local biasing in the physical, non-Gaussian density field.

In accordance with local biasing (see e.g., [3,13]), we associate peaks with positions where the smoothed density field $\delta_{R}$ is larger than a threshold $\nu \sigma_{R}$ (hence, for $\nu=3$ we are selecting $3 \sigma$ peaks of the smoothed density field). The average number density of such peaks is then given by

$$
n_{\mathrm{pk}}=\left\langle\Theta\left[\delta_{R}(\mathbf{x})-\nu \sigma_{R}\right]\right\rangle,
$$

where $\Theta$ is the Heaviside function, and $\langle\cdot\rangle$ here denotes the ensemble average. Similarly, the correlation function of peaks is given by (using the same shorthand notation as above)

$$
\xi_{\mathrm{pk}}(r)=\frac{\left\langle\Theta\left[\delta_{1}-\nu \sigma_{R}\right] \Theta\left[\delta_{2}-\nu \sigma_{R}\right]\right\rangle}{n_{\mathrm{pk}}^{2}}-1 .
$$

We are interested in the pairwise velocity of peaks along the separation $r$. We can write the pair-weighted probability distribution for the two velocities $\bar{u}_{r 1}, \bar{u}_{r 2}$ as

$$
\begin{aligned}
\mathcal{P}\left(\bar{u}_{r 1}, \bar{u}_{r 2} ; \mathbf{x}_{1}, \mathbf{x}_{2}\right)= & n_{\mathrm{pk}}^{-2} \times\left\langle\delta_{D}\left(\hat{\mathbf{r}} \cdot \mathbf{u}_{1}-\bar{u}_{r 1}\right)\right. \\
& \times \delta_{D}\left(\hat{\mathbf{r}} \cdot \mathbf{u}_{2}-\bar{u}_{r 2}\right) \Theta\left[\delta_{1}-\nu \sigma_{R}\right] \\
& \left.\times \Theta\left[\delta_{2}-\nu \sigma_{R}\right]\right\rangle .
\end{aligned}
$$

Here, $\delta_{D}$ denotes the Dirac $\delta$ function. Note that by construction, the peak correlation function Eq. (29) is recovered through

$$
1+\xi_{\mathrm{pk}}(r)=\int \mathrm{d} \bar{u}_{r 1} \int \mathrm{d} \bar{u}_{r 2} \mathcal{P}\left(\bar{u}_{r 2}, \bar{u}_{r 2} ; \mathbf{x}_{1}, \mathbf{x}_{2}\right) .
$$

Using Eq. (30), the volume-weighted distribution $\mathcal{P}_{\delta u}$ of the pairwise peak velocity $\delta u \equiv \bar{u}_{r 2}-\bar{u}_{r 1}$ at a given separation is obtained as [see also Eq. (21)]

$\mathcal{P}_{\delta u}\left(u ; \mathbf{x}_{1}, \mathbf{x}_{2}\right)=\frac{1}{1+\xi_{\mathrm{pk}}(r)} \int \mathrm{d} \bar{u}_{r 1} \mathcal{P}\left(\bar{u}_{r 1}, u+\bar{u}_{r 1} ; \mathbf{x}_{1}, \mathbf{x}_{2}\right)$. 
We now write the ensemble average of any functional $\mathcal{F}[\mathbf{u}]$ of the velocity field as a functional integral over all possible realizations of the (linear) velocity field $\mathbf{u}(\mathbf{x})$ :

$$
\langle\mathcal{F}[\mathbf{u}]\rangle=\int \mathcal{D}[\mathbf{u}(\mathbf{x})] \mathcal{P}[\mathbf{u}] \mathcal{F}[\mathbf{u}]
$$

Here, $\mathcal{D}[\mathbf{u}]$ is the functional measure, and $\mathcal{P}[\mathbf{u}]$ is the probability distribution of the continuous velocity field. We make use of a result of statistical field theory (see e.g., [1315] for related applications), expressing the partition function $Z[\mathbf{J}]$ in terms of higher-order (connected) correlation functions:

$$
\begin{aligned}
Z[\mathbf{J}]= & \int \mathcal{D}[\mathbf{u}(\mathbf{x})] \mathcal{P}[\mathbf{u}] \exp \left\{i \int \mathrm{d}^{3} \mathbf{x} \mathbf{J}(\mathbf{x}) \cdot \mathbf{u}(\mathbf{x})\right\}, \\
= & \exp \left\{\sum_{n=2}^{\infty} \frac{i^{n}}{n !} \int \mathrm{d}^{3} \mathbf{x}_{1} \ldots \int \mathrm{d}^{3} \mathbf{x}_{n} \xi_{i_{1} \ldots i_{n}}^{(n)}\left(\mathbf{x}_{1}, \ldots \mathbf{x}_{n}\right)\right. \\
& \left.\times J^{i_{1}}\left(\mathbf{x}_{1}\right) \ldots J^{i_{n}}\left(\mathbf{x}_{n}\right)\right\} .
\end{aligned}
$$

Here,

$$
\xi_{i_{1} \ldots i_{n}}^{(n)}\left(\mathbf{x}_{1}, \ldots \mathbf{x}_{n}\right)=\left\langle u_{i_{1}}\left(\mathbf{x}_{1}\right) \ldots u_{i_{n}}\left(\mathbf{x}_{n}\right)\right\rangle_{\text {con }}
$$

is the connected $n$-point correlation function of the $u n$ smoothed velocity field. We can now use $\delta_{R}=-\nabla \mathbf{u}_{R}$ together with Eq. (34) to obtain the velocity probability distribution of peaks defined in Eq. (30), in close analogy to the derivation for density correlations presented in [13]. This calculation is detailed in Appendix B.

We only keep terms linear in the correlations, an approximation valid on large scales. Once we have the expression for $\mathcal{P}_{\delta u}$ [Eq. (32)], it is straightforward to obtain the moments of the relative peak velocity $\delta u$ via

$$
\left\langle\delta u^{n}\right\rangle_{\mathrm{pk}}(r)=\int \mathrm{d} \delta u \delta u^{n} \mathcal{P}_{\delta u}(\delta u ; r) .
$$

Note that the central and noncentral moments agree to first order in the correlations (since $\langle\delta u\rangle_{\mathrm{pk}}$ is already first order). We then obtain (Appendix B):

$$
\begin{gathered}
\langle\delta u\rangle_{\mathrm{pk}}=2 b_{L} \xi_{R}^{u \delta}(r)+b_{L}^{2}\left[\xi_{R}^{\delta \delta u}(r)-2 \xi_{R}^{\delta u \delta}(r)\right], \\
\left\langle\delta u^{2}\right\rangle_{\mathrm{pk}}=2\left[\sigma_{u}^{2}+\xi_{R}^{u u}(r)+b_{L} \xi_{R}^{u u \delta}(0)+b_{L} \xi_{R}^{u u \delta}(r)\right. \\
\left.-2 b_{L} \xi_{R}^{\delta u u}(r)\right] \\
\left\langle\delta u^{3}\right\rangle_{\mathrm{pk}}=6 \xi_{R}^{u u u}(r) \\
\left\langle\delta u^{4}\right\rangle_{\mathrm{pk}}-3\left\langle\delta u^{2}\right\rangle_{\mathrm{pk}}^{2}=0
\end{gathered}
$$

These moments agree with those derived in Sec. III A. Note that there is no connected fourth moment (kurtosis) at this order, since we have assumed no primordial trispectrum. The fact that the moments of the peak velocity distribution agree with those derived in Sec. III A shows that no largescale statistical velocity bias of peaks is induced by nonGaussianity.

\section{DISTRIBUTION OF RELATIVE VELOCITIES}

Figure 1 shows the moments of $\delta u$ in the Gaussian and non-Gaussian case as function of the separation $r$. Here, we have assumed local non-Gaussianity with $f_{\mathrm{NL}}=100$, and halos with $10^{13} M_{\odot} / h$ at $z=0$, corresponding to a smoothing scale of $R=3.1 \mathrm{Mpc} / h$ and a Lagrangian bias of $b_{L}=0.88$.

The mean streaming velocity of halos $\langle\delta u\rangle_{\mathrm{pk}}$ is always negative, since overdense regions have a net infall velocity. It is clearly enhanced on large scales by non-Gaussianity. This is mainly due to the term $b_{L}^{2} \xi_{R}^{\delta \delta u}$, which corresponds to the density-weighted analog of $\xi_{R}^{u \delta}$ and is negative for positive $f_{\mathrm{NL}}$, i.e. for a positively skewed density field. The cause of this is the same as that which leads to a strong enhancement of the halo correlation function on large scales in the presence of local non-Gaussianity: in this

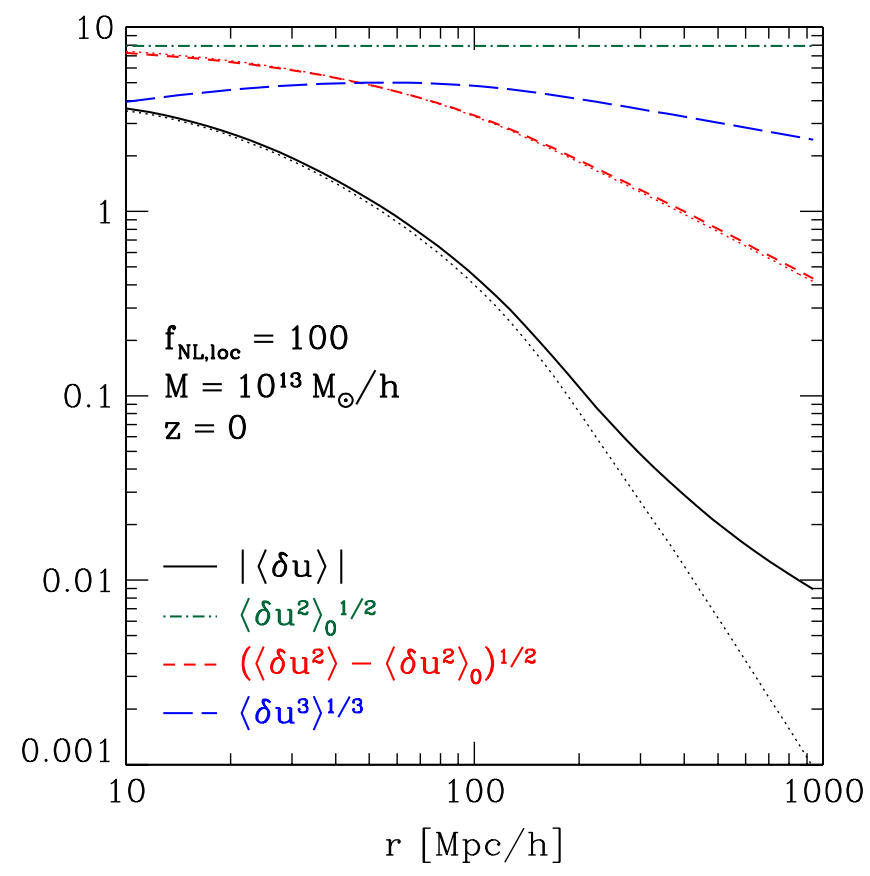

FIG. 1 (color online). Moments of the relative velocity distribution $\mathcal{P}_{\delta u}$ as function of the separation $r$. The thick lines show the moments for a cosmology with primordial non-Gaussianity of the local type $\left(f_{\mathrm{NL}}=100\right)$, while the thin dotted lines show those for vanishing non-Gaussianity. We have separated the variance into a scale-independent part $\left\langle\delta u^{2}\right\rangle_{0}$ and a scaledependent part (see text). In all cases we assumed halos with $M=10^{13} M_{\odot} / h$ at $z=0$ (smoothing scale $R \approx 3.1 \mathrm{Mpc} / h$, $\left.b_{L}=0.88\right)$. 
model, long-wavelength modes of the potential are coupled to short wavelength modes of the density field, leading to a strong scale-dependent increase in the large-scale correlations of halos.

On the other hand, the effect on the variance $\left\langle\delta u^{2}\right\rangle_{\mathrm{pk}}$ is very small. In Fig. 1, we have split the variance into a scaledependent and scale-independent part, and denoted the latter by $\left\langle\delta u^{2}\right\rangle_{0}$. The scale-independent standard deviation of $\delta u$ is $7.9 \mathrm{Mpc} / h$ for the adopted smoothing scale and $f_{\mathrm{NL}}=100$, while it is $8.1 \mathrm{Mpc} / h$ in the Gaussian case, a difference of only $3 \%$. Thus, the effect of non-Gaussianity on the scale-independent as well as scale-dependent part (Fig. 1) of the variance is small. Note that the non-Gaussian terms in the variance are proportional to the bias $b_{L}$.

Finally, the third moment of $\delta u$ is significant. The skewness, defined as $\left\langle\delta u^{3}\right\rangle_{\mathrm{pk}} /\left\langle\delta u^{2}\right\rangle_{\mathrm{pk}}^{3 / 2}$, is between 0.1 and 0.2 on a wide range of scales. The skewness which quantifies how strongly non-Gaussian the velocity distribution is, depends only weakly on the halo mass, through the smoothing scale $R$, and redshift. Since $\left\langle\delta u^{2}\right\rangle_{\mathrm{pk}}$ slowly declines with increasing smoothing scale while $\left\langle\delta u^{3}\right\rangle_{\mathrm{pk}}$ is essentially independent of $R$, the skewness is slightly larger for higher mass halos, e.g. $10 \%$ higher for $10^{14} M_{\odot} / h$ halos compared to $10^{13} M_{\odot} / h$. Note that for typical choices of the smoothing scale, there will be a significant nonlinear correction to the variance $\left\langle\delta u^{2}\right\rangle_{\mathrm{pk}}$.

Further, it is important to note that the skewness is nonzero for other bispectrum shapes as well. For example, a bispectrum of the equilateral type (see Appendix A), again with $f_{\mathrm{NL}}=100$, yields a skewness of about 0.06 at $r=100 \mathrm{Mpc} / h$, only a factor of 3 smaller than the skewness obtained in the local model. On the other hand, the power spectrum of halos only receives a scale-independent correction in the equilateral model ([16], Sec. V). Thus, the distribution of relative velocities could be an interesting avenue to test especially those models of non-Gaussianity which can only be weakly constrained from the clustering of halos.

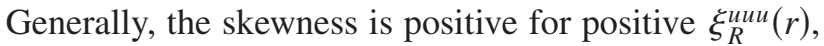
which is usually the case when $\xi_{R}^{\delta \delta \delta}$ is positive (i.e., for positive $f_{\mathrm{NL}}$ ). At first this might seem counterintuitive, since for a positively skewed density field there are more overdense than underdense regions. Hence, one expects that velocities projected on $\hat{r}$ are preferentially "inward," i.e., negative. However, this is only true for densityweighted velocity correlations, such as $\xi_{R}^{\delta \delta u}$ which is indeed negative. $\xi_{R}^{\text {uиu }}=\left\langle u_{1 r}^{2} u_{2 r}\right\rangle$ on the other hand is a velocity-weighted velocity correlation. $\xi_{R}^{u и u}>0$ thus means that high-velocity regions on average move apart (while there is no such net motion in the Gaussian case). High-velocity regions correspond to infall regions of overdensities, so $\xi_{R}^{u и u}$ corresponds to enhanced infall motions. This is not unexpected for a density field with more highdensity regions. Of course, the converse holds for a negatively skewed density field $\left(f_{\mathrm{NL}}<0\right)$.
It is also interesting to look at the full distribution of $\mathcal{P}_{\delta u}(\delta u)$. Since by assumption the fourth and higher velocity moments are small (and in fact all moments other than the variance are small on large scales), we can employ the Edgeworth expansion (e.g., $[15,17])$ :

$$
\begin{aligned}
\mathcal{P}_{\delta u}\left(\delta u ; \mathbf{x}_{1}, \mathbf{x}_{2}\right)= & \left\{1+\frac{\langle\delta u\rangle_{\mathrm{pk}}}{2 \sigma_{u}^{2}} \delta u+\frac{\left\langle\delta u^{2}\right\rangle_{\mathrm{pk}}}{8 \sigma_{u}^{2}}\left(\frac{\delta u^{2}}{\sigma_{u}^{2}}-2\right)\right. \\
& \left.+\frac{\left\langle\delta u^{3}\right\rangle_{\mathrm{pk}}}{12 \sigma_{u}^{3}} \frac{\delta u}{4 \sigma_{u}}\left(\frac{\delta u^{2}}{\sigma_{u}^{2}}-6\right)\right\} \frac{1}{\sqrt{4 \pi \sigma_{u}^{2}}} \\
& \times \exp \left(-\frac{\delta u^{2}}{4 \sigma_{u}^{2}}\right) .
\end{aligned}
$$

We also arrive at this expression directly using the statistical approach of Appendix B. The distribution of the relative velocity $\delta u$ at a separation of $100 \mathrm{Mpc} / h$ is shown in Fig. 2 in the Gaussian and non-Gaussian case, for the same halos and local $f_{\mathrm{NL}}=100$ as in Fig. 1. The effect of non-Gaussianity is clearly visible as skewing the distribution towards positive velocities, as expected from the positive third moment. Correspondingly, a negative $f_{\mathrm{NL}}$ would lead to a velocity distribution skewed towards negative relative velocities. Again, these results are only weakly dependent on halo mass and redshift. Furthermore, the relative velocity distribution of the same halos in an equilateral model with $f_{\mathrm{NL}} \approx 300$ looks very similar to the local model result shown in Fig. 2.

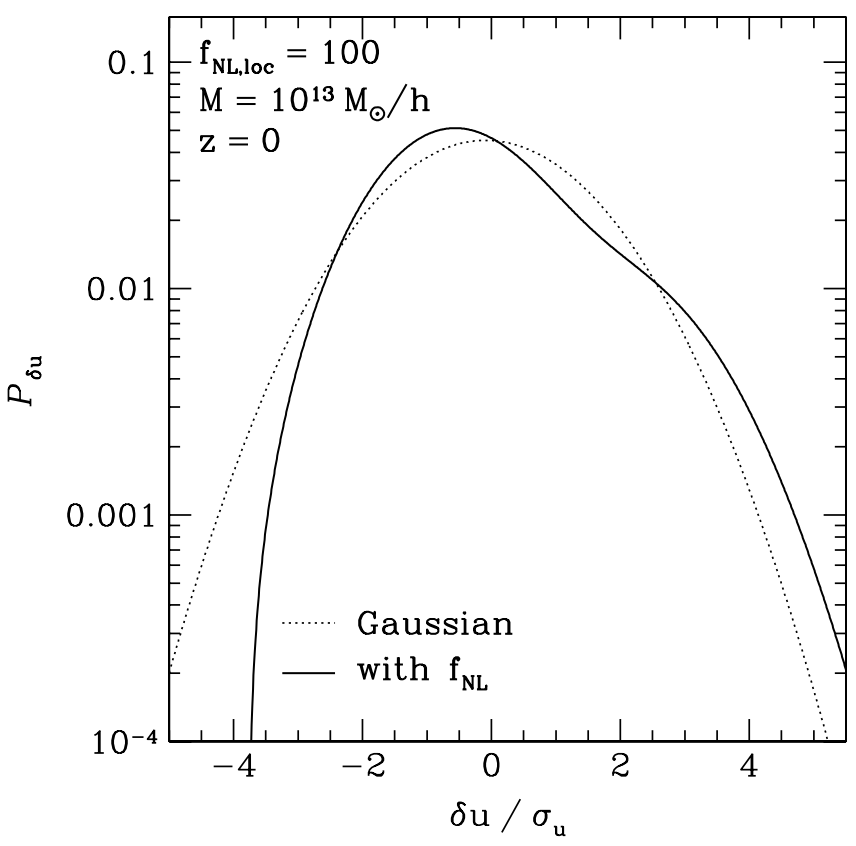

FIG. 2 (color online). Distribution of the relative velocity $\delta u$ for a separation $r=100 \mathrm{Mpc} / h$ in the non-Gaussian (solid) and Gaussian (dotted) case, for the same parameters and halos as in Fig. 1. Note that an equilateral model with $f_{\mathrm{NL}} \approx 300$ would lead to a result very similar to the local model shown. 
Recently, observational studies have found evidence for larger bulk flow motions than expected in $\Lambda \mathrm{CDM}$. These studies have used galaxy peculiar velocity surveys [18], the kinetic Sunyaev-Zeldovich effect [19], and the estimated initial relative velocity of the merging halos in the Bullet cluster [20]. Figure 2 shows that even a moderate primordial non-Gaussianity that is still allowed at the $2 \sigma$ level can significantly alter the tails of the velocity distribution. Thus an obvious question is whether the existence of primordial non-Gaussianity can alleviate the tension between the reported bulk flow observations and the $\Lambda$ CDM scenario. Unfortunately, it is not straightforward to relate our predictions of linear perturbation theory in the large-scale limit to these observational results. Thus, we do not attempt any quantitative comparison here.

\section{MATTER AND PEAK CORRELATIONS IN REDSHIFT SPACE}

One of the most important observational effects of largescale velocities is their impact on correlations of tracers such as galaxies, clusters, or the Lyman- $\alpha$ forest, through redshift distortions. Positions are measured in redshift space, whose coordinates $\mathbf{s}$ are related to real-space coordinates $\mathbf{x}$ by a shift along the line-of-sight direction:

$$
\mathbf{s}=\mathbf{x}+f u_{z} \hat{\mathbf{z}} .
$$

Here, we have chosen the line of sight to be along the $z$ axis. Throughout, we will work in the flat sky limit. The statistics of the density field (of matter or peaks) in redshift space can be determined using the real-space statistics of the density and velocity fields together with the mapping Eq. (43).

We start with the normalized distribution $\mathcal{P}_{\delta u}(\delta u, r)$. The correlation function in redshift space $\xi_{s}$ can then be written as a convolution by the velocity difference distribution $\mathcal{P}_{\delta u}(\delta u ; r)$ as $[2,21]$

$$
\begin{aligned}
1+\xi_{s}\left(s_{z}, \mathbf{s}_{\perp}\right) & =\int \mathrm{d} \delta u[1+\xi(r(\delta u))] \mathcal{P}_{\delta u}(\delta u ; r), \\
r(\delta u) & =\sqrt{\mathbf{s}_{\perp}^{2}+\left(s_{z}-\mu f \delta u\right)^{2}},
\end{aligned}
$$

where the factor $\mu=r_{z} / r$ multiplies $\delta u$, the total velocity along $r$, to obtain the velocity along the line of sight. This expression is exact in the flat sky limit. On large scales, the displacements from real to redshift space are much smaller than the separation $r$, and we can expand $\xi$ as well as $\mathcal{P}_{\delta u}$ around $r_{z}=s_{z}$. Generalizing expressions given in, e.g., [21], we obtain

$$
\begin{aligned}
1+\xi_{s}\left(s_{z}, \mathbf{s}_{\perp}\right)= & \sum_{n, m=0}^{\infty} \frac{(-\mu f)^{n+m}}{n ! m !} \frac{d^{n}}{d s_{z}^{n}}(1+\xi(s)) \frac{d^{m}}{d s_{z}^{m}} \\
& \times\left\langle\delta u^{n+m}\right\rangle(s),
\end{aligned}
$$

where now $\mu=s_{z} / s$. Further, we keep only those terms which are first order in large-scale correlations. This gives

$$
\begin{aligned}
\xi_{s}\left(s_{z}, \mathbf{s}_{\perp}\right)= & \xi(s)-f \mu\langle\delta u\rangle^{\prime}(s)+\frac{f^{2} \mu^{2}}{2}\left\langle\delta u^{2}\right\rangle^{\prime \prime}(s) \\
& -\frac{f^{3} \mu^{3}}{6}\left\langle\delta u^{3}\right\rangle^{\prime \prime \prime}(s)+\frac{f^{2} \mu^{4}}{2}\left\langle\delta u^{2}\right\rangle(s) \xi^{\prime \prime}(s) \\
& -\frac{f^{3} \mu^{6}}{6}\left\langle\delta u^{3}\right\rangle(s) \xi^{\prime \prime \prime}(s),
\end{aligned}
$$

where primes denote derivatives with respect to $s_{z}$. The last two terms are formally second order, however the moments of the velocity $\left\langle\delta u^{m}\right\rangle$ can be significant even on large scales due to small-scale motion. However, we verified that the last, non-Gaussian term is negligible compared to the other terms. Since the Gaussian quadratic term is quite small on large scales as well, we will neglect those two terms in the following.

We can now Fourier transform Eq. (46) term by term, noting that every derivative with respect to $s_{z}$ brings down a power of $i k_{z}=i \mu k$. Defining the Fourier transform $\left\langle\widetilde{\delta u^{n}}\right\rangle(k)$ of the $n$th velocity moment (in the flat sky limit) via

$$
\left\langle\delta u^{n}\right\rangle(r)=\int \frac{\mathrm{d}^{3} \mathbf{k}}{(2 \pi)^{3}} e^{i \mathbf{k} \cdot \mathbf{r}}\left\langle\widetilde{\delta u^{n}}\right\rangle(k),
$$

we can Fourier transform Eq. (46) to obtain an expression for the redshift-space power spectrum $P_{s}(k, \mu)$ :

$$
\begin{aligned}
P_{s}(k, \mu)= & P(k)-f i \mu^{2} k\langle\widetilde{\delta u}\rangle(k)+\frac{f^{2}}{2}\left(-\mu^{4} k^{2}\right)\left\langle\widetilde{\delta u^{2}}(k)\right\rangle \\
& -\frac{f^{3}}{6} \mu^{6}(i k)^{3}\left\langle\widetilde{\delta u^{3}}\right\rangle(k),
\end{aligned}
$$

where $\mu=k_{z} / k$. Note that this expression applies to both peaks and matter. With the results of Sec. III, it is then straightforward to write down the redshift-space peak power spectrum Eq. (48) in the presence of nonGaussianities. Again, these results apply to bispectra of arbitrary shape and scale dependence.

Fourier transforming the $\delta u$ moments given by Eqs. (23), (24), and (27) is easily done by replacing the correlations $\xi^{l m n}(r)$ with $\mathcal{P}^{l m n}(k)$. The full expression of the large-scale limit then reads

$$
\begin{aligned}
P_{s}(k, \mu)= & b_{L}^{2} P_{R}(k)+b_{L}^{3} \mathcal{P}^{\delta \delta \delta}-f \mu^{2} i k\left[2 b_{L}(i k)^{-1} P_{R}(k)\right. \\
& \left.+b_{L}^{2}\left(i \mathcal{P}^{\delta \delta u}(k)-2 i \mathcal{P}^{\delta u \delta}(k)\right)\right] \\
& -\frac{f^{2}}{2} \mu^{4} k^{2}\left[2(i k)^{-2} P_{R}(k)-2 b_{L} \mathcal{P}^{u и \delta}(k)\right. \\
& \left.+4 b_{L} \mathcal{P}^{\delta и u}(k)\right]-\frac{f^{3}}{6} \mu^{6}(i k)^{3} 6 i^{3} \mathcal{P}^{\text {иии }(k) .}
\end{aligned}
$$

The first line contains the real-space peak power spectrum including the well-known non-Gaussian contribution ([6], see Eq. (B30) in Appendix B). Note that in Eulerian space, $b_{L}^{3}$ is to be replaced by $b_{1} b_{2}$. Using $\mathcal{P}^{l m u}=\mathcal{P}^{l m \delta} / k$, which follows from Eq. (19), we get 


$$
\begin{aligned}
P_{s}(k, \mu)= & b_{L}^{2} P_{R}(k)+2 b_{L} f \mu^{2} P_{R}(k)+f^{2} \mu^{4} P_{R}(k) \\
& +b_{L}^{3} \mathcal{P}^{\delta \delta \delta}(k)+f \mu^{2} b_{L}^{2} \mathcal{P}^{\delta \delta \delta}(k) \\
& -2 f \mu^{2} b_{L}^{2} k \mathcal{P}^{\delta и \delta}(k) \\
& +f^{2} \mu^{4} b_{L} k^{2}\left[\mathcal{P}^{\text {ии } \delta}(k)-2 \mathcal{P}^{\delta и и ~}(k)\right] \\
& +f^{3} \mu^{6} k^{3} \mathcal{P}^{\text {иии }}(k) .
\end{aligned}
$$

The terms in the first line constitute the usual Gaussian power spectrum of biased peaks in redshift space. Following convention [5-7], we can write the first term in the second line as a non-Gaussian correction $\Delta b_{L}$ to the Gaussian halo bias (see, e.g., [6]):

$$
\frac{\Delta b_{L}}{b_{L}}=\frac{1}{2} b_{L} \frac{\mathcal{P}^{\delta \delta \delta}(k)}{P_{R}(k)} \text {. }
$$

Thus, if we include this non-Gaussian bias and neglect the terms in the last three lines above, the Kaiser formula Eq. (1) is still valid in the presence of large-scale nonGaussianity:

$$
P_{s}^{\mathrm{Kaiser}}(k, \mu)=\left(1+\frac{f \mu^{2}}{b_{L}+\Delta b_{L}}\right)^{2}\left(b_{L}+\Delta b_{L}\right)^{2} P_{R}(k) .
$$

However, there are additional terms which we will denote as $P_{s}^{\mathrm{NG}}$ :

$$
\begin{aligned}
P_{s}^{\mathrm{NG}}(k, \mu)= & -2 k \mathcal{P}^{\delta u \delta}(k)\left[f \mu^{2} b_{L}^{2}+f^{2} \mu^{4} b_{L}\right] \\
& +k^{2} \mathcal{P}^{u и \delta}(k)\left[f^{2} \mu^{4} b_{L}+f^{3} \mu^{6}\right] .
\end{aligned}
$$

Corrections of order $f_{\mathrm{NL}}$ to the Kaiser formula were also found by [22] for the variance of the density field in redshift space. Figure 3 shows the remaining terms which violate the Kaiser formula in the presence of nonGaussianity on large scales. Comparing them to the realspace contribution $\mathcal{P}^{\delta \delta \delta}$, it is clear that these terms are far subdominant. However, they become increasingly important towards smaller scales. Since the linear perturbation theory used here begins to break down on scales $k \gtrsim$ $0.05 h / \mathrm{Mpc}$, it will be necessary to extend the perturbative calculation to higher order or to measure the effect in $\mathrm{N}$-body simulations. In the adopted cosmology, the nonlinear scale for matter is $k_{\mathrm{nl}} \approx 0.27 \mathrm{~h} / \mathrm{Mpc}$, where $k_{\mathrm{nl}}$ is defined through $\Delta^{2}\left(k_{\mathrm{nl}}\right)=k_{\mathrm{nl}}^{3} P\left(k_{\mathrm{nl}}\right) / 2 \pi^{2}=1$. Note that $k_{\mathrm{nl}}$ will be somewhat smaller for massive halos whose power spectrum is enhanced by $b^{2}$.

Figure 4 shows the same results for the equilateral and folded bispectrum shapes. The overall corrections to the Gaussian power spectrum are much smaller, since these bispectrum shapes are suppressed in the squeezed limit. The relative importance of the additional terms from Eq. (53) is qualitatively similar to the local case. Note that for all bispectra, the relative significance of these terms compared with the real-space contribution $\mathcal{P}^{\delta \delta \delta}$ is independent of $f_{\mathrm{NL}}$ at leading order.

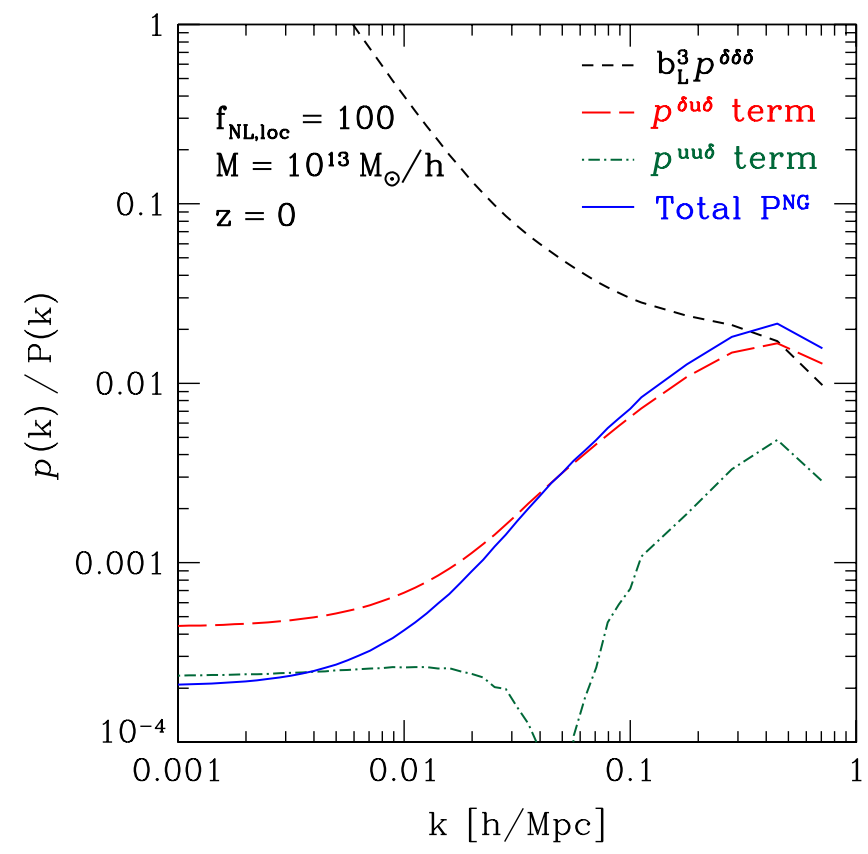

FIG. 3 (color online). The terms of Eq. (53) which contribute to the redshift-space power spectrum and are not captured by the Kaiser formula, as function of $k$. We also show the leading realspace term $\mathcal{P}^{\delta \delta \delta}(k)$ for comparison. All terms are for $10^{13} M_{\odot} / h$ halos at $z=0$ and local non-Gaussianity with $f_{\mathrm{NL}}=100$. We have set $\mu=1$ and divided all terms by the matter power spectrum. Note that nonlinear corrections to these linear predictions are expected above $k \sim 0.05 h / \mathrm{Mpc}$.

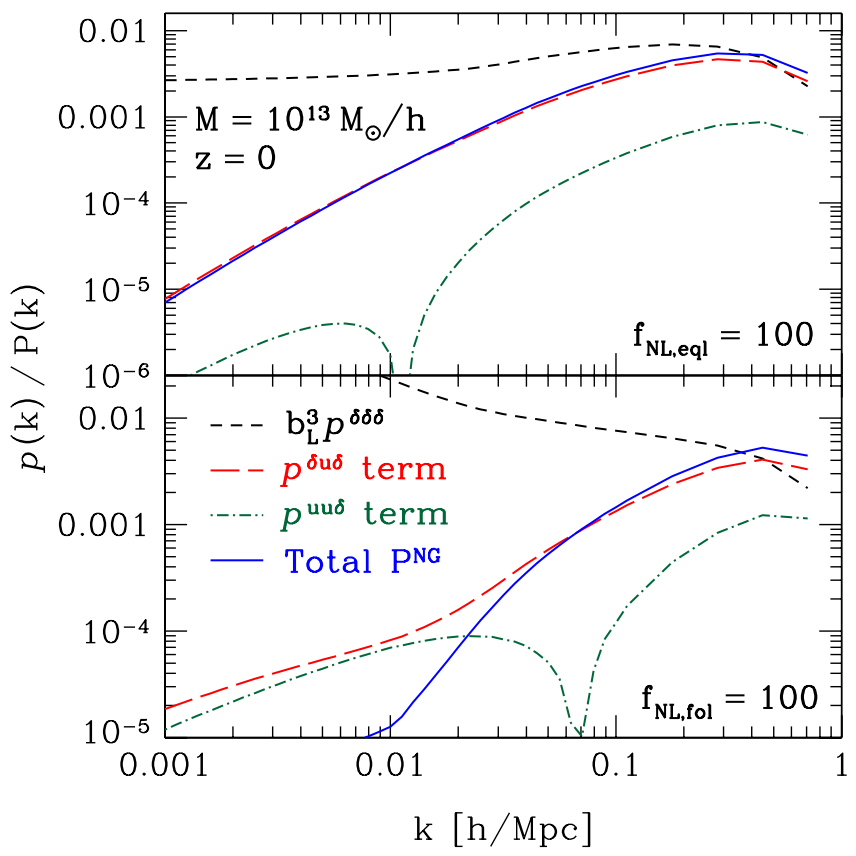

FIG. 4 (color online). Same as Fig. 3, but for equilateral (top panel) and folded (bottom panel) primordial bispectra with $f_{\mathrm{NL}}=100$ (see Appendix A). 


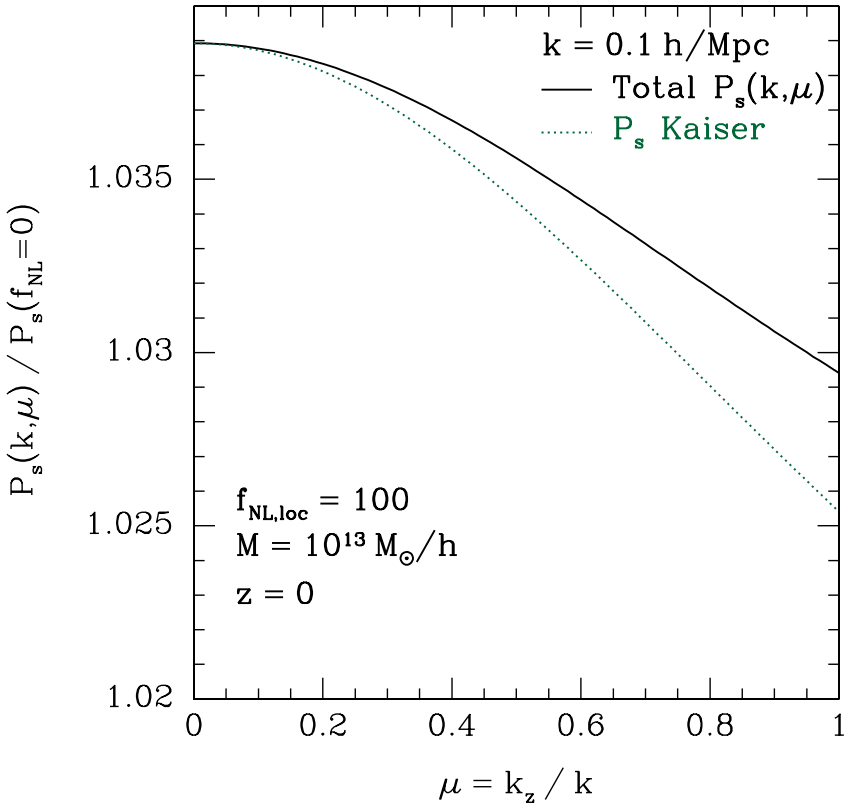

FIG. 5 (color online). Ratio of the redshift-space power spectrum with primordial non-Gaussianity $\left(f_{\mathrm{NL}}=100\right)$ to that with Gaussian initial conditions as function of $\mu$ at $k=0.1 \mathrm{~h} / \mathrm{Mpc}$. Again, results are shown for $10^{13} M_{\odot} / h$ halos at $z=0$. Shown is the actual ratio including all terms (Eqs. (52) and (53)), and the ratio one obtains from the Kaiser formula (Eq. (52) only). Note that this is mainly illustrative, since we are showing linear predictions at a scale already in the quasilinear regime (nonlinear correction from HALOFIT 5\%).

As an example illustrating the effect on the redshiftspace power spectrum, Fig. 5 shows $P_{s}(k, \mu)$ at $k=$ $0.1 \mathrm{~h} / \mathrm{Mpc}$ as function of $\mu$, when using the Kaiser formula Eq. (52) and when including all terms. At these small scales, the difference is clearly noticeable. This is only a rough illustration however, since in reality nonlinear corrections will contribute significantly at that scale: the nonlinear power spectrum (from HALOFIT [23]) $k=0.1 h / \mathrm{Mpc}$ differs by $\sim 5 \%$ from the linear $P(k)$. Still, we expect that the corrections to the Kaiser formula will remain at least at the percent level for $k \gtrsim 0.1 \mathrm{~h} / \mathrm{Mpc}$. That would make them possibly relevant for upcoming spectroscopic surveys such as BOSS and HETDEX, and certainly for future space missions such as JDEM and EUCLID, which are expected to constrain redshift distortions at the sub-percent level $[24,25]$.

Again, these results do not depend sensitively on halo mass and redshift: if, for example, we increase the halo mass, there is a partial cancellation between the larger smoothing scale and the increased halo bias, leaving the relative size of the terms in Eq. (53) roughly unchanged. For $z>0, f$ increases (asymptoting to 1 at very high redshifts), but this is compensated by larger biases at fixed mass.

Finally, we note that the last term in Eq. (53), which comes from the skewness of the velocity distribution, also contributes to the redshift-space power spectrum of an unbiased tracer, for example, matter itself (see also [22]). This is in contrast to the linear matter power spectrum in real space, which only receives a much smaller four-point correction of order $f_{\mathrm{NL}}^{2}$.

The reasons that the additional terms are so much smaller are first, that the dominant effect on the mean streaming of halos is taken into account by the mixed density-velocity part of the Kaiser formula (when including the scale-dependent bias correction); and second, that the third moment of $\delta u$, though significantly changing the distribution of $\delta u$, only affects the correlation function via three derivatives [Eq. (46)], or three powers of $k$ in the case of the power spectrum. This strongly suppresses its contribution on large scales. Note that this is specific to the power spectrum, and a larger effect of $\xi_{R}^{u u u}$ is expected, e.g., for the bispectrum in redshift space.

\section{CONCLUSIONS}

In this paper, we have studied the large-scale motions of peaks in the density field in the presence of primordial nonGaussianity. In the high-peak regime we are interested in, peaks can be identified with dark matter halos and visible structures such as galaxies and clusters. We have derived the statistics of the relative velocity of halo pairs in two different approaches based on a local biasing scheme. Note that the bias is local in the physical, non-Gaussian density field. The first, simpler and more accessible approach assumes that on large scales, halo velocities follow those of the total matter. Given linear and quadratic bias parameters, it is then straightforward to calculate the moments of the relative velocity distribution. In the second approach (see Appendix B), no assumptions are made apart from local biasing and the large-scale, small-correlation limit. Both approaches agree, showing that the assumption of statistically unbiased halo velocities is consistent on large scales even in the presence of non-Gaussianity.

Interestingly, the presence of nonzero three-point correlations on large scales leads to significant changes in the velocity distribution of halos, and matter itself. For a positive $f_{\mathrm{NL}}$, the mean streaming velocity is significantly enhanced on large scales, and a nonzero third moment leads to a positive skewness of the velocity difference distribution. In the local model, the skewness $\left\langle\delta u^{3}\right\rangle_{\mathrm{pk}} /\left\langle\delta u^{2}\right\rangle_{\mathrm{pk}}^{3 / 2}$ is of order $(0.1-0.2) \times\left(f_{\mathrm{NL}} / 100\right)$ on a wide range of scales. Even in the equilateral model, which has little effect on the halo power spectrum, the skewness still reaches 0.06 for the same $f_{\mathrm{NL}}$. This suggests that the velocity difference distribution can serve as an interesting probe of non-Gaussianity (as already pointed out by [10]). Furthermore, these findings could be of relevance to recent observational reports of significantly higher velocities than expected in the $\Lambda \mathrm{CDM}$ (or more generally, General Relativity + Dark Energy) scenario [18-20]. In order to evaluate this quantitatively however, one has to 
take into account nonlinear corrections, via perturbation theory and/or simulations.

Our second result is an expression for the redshift-space power spectrum of halos (or matter) in the presence of nonGaussianity. The well-known Kaiser formula relating realand redshift-space power spectra, extended by the scaledependent halo bias (see, e.g., [4]), receives non-Gaussian corrections which become relevant on small scales. Note that through these corrections, the redshift-space power spectrum measured at a given scale as function of lineof-sight angle in principle allows for a direct measurement of non-Gaussianity from a given tracer population, without any reference to the underlying matter power spectrum (in the Kaiser formula at a fixed scale, the non-Gaussian effects are perfectly degenerate with the galaxy bias). Furthermore, the redshift-space power spectrum of matter itself receives corrections of order $f_{\mathrm{NL}}$ from nonGaussianity, which are not present in real space.

The non-Gaussian corrections to the Kaiser formula lead to the interesting question of whether there are degeneracies of the effects of non-Gaussianity with other parameters measured from redshift-space distortions, such as dark energy parameters or consistency tests of general relativity $[26,27]$. The severity of the degeneracies will depend on the level of allowed non-Gaussianity. While the local model is likely to be constrained tightly in the near future, other models such as the equilateral model are much less constrained, but can still be lead to noticeable effects in redshift space (Fig. 4).

Finally, we expect that non-Gaussian effects on halo velocities will have an even larger impact on the bispectrum of halos in redshift space. Again, these questions deserve more study via higher-order perturbation theory as well as $N$-body simulations.

\section{ACKNOWLEDGMENTS}

I am grateful to V. Desjacques for very helpful discussions and providing important insights in the initial stages of this project. Thanks also goes to U. Seljak and the Institute for Theoretical Physics at the University of Zürich for their hospitality. Further, I would like to thank M. Kamionkowski, N. Afshordi, T. Baldauf, N. Dalal, U. Seljak, R. Scoccimarro, and especially O. Doré for enlightening discussions. This work was supported by the Gordon and Betty Moore Foundation at Caltech.

\section{APPENDIX A: MATTER BISPECTRA FROM PRIMORDIAL NON-GAUSSIANITY}

Primordial non-Gaussianity is most easily characterized by the bispectrum of the primordial curvature perturbations. Different shapes, i.e., configuration dependences, have been proposed in the literature. Since we work in the subhorizon regime at late times, we phrase nonGaussianity in terms of the potential $\Phi$ at early times, which can be directly related to the primordial perturbations. The bispectrum $B_{\Phi}$ at a fixed redshift is defined by

$$
\begin{aligned}
\left\langle\Phi\left(\mathbf{k}_{1}\right) \Phi\left(\mathbf{k}_{2}\right) \Phi\left(\mathbf{k}_{3}\right)\right\rangle= & (2 \pi)^{3} \delta_{D}\left(\mathbf{k}_{1}+\mathbf{k}_{2}+\mathbf{k}_{3}\right) \\
& \times B_{\Phi}\left(k_{1}, k_{2}, k_{3}\right) .
\end{aligned}
$$

Throughout, we take $B_{\Phi}$ to be defined at last scattering ("CMB convention", $z_{*} \approx 1100$ ). The bispectrum corresponding to local non-Gaussianity of the type Eq. (3) is

$$
B_{\Phi}^{\text {loc }}\left(k_{1}, k_{2}, k_{3}\right)=2 f_{\mathrm{NL}}\left(P_{\Phi}\left(k_{1}\right) P_{\Phi}\left(k_{2}\right)+2 \text { perm }\right) \text {, }
$$

where $P_{\Phi}$ is the power spectrum of $\Phi$ at $z_{*}$. The bispectra corresponding to equilateral and (en)folded types are given by

$$
\begin{aligned}
B_{\Phi}^{\mathrm{eql}}= & 6 f_{\mathrm{NL}}\left[-P_{1} P_{2}-2 \text { perm }-2\left(P_{1} P_{2} P_{3}\right)^{2 / 3}\right. \\
& \left.+P_{1}^{1 / 3} P_{2}^{2 / 3} P_{3}+5 \text { perm }\right], \\
B_{\Phi}^{\mathrm{fol}}= & 6 f_{\mathrm{NL}}\left[P_{1} P_{2}+2 \text { perm }+3\left(P_{1} P_{2} P_{3}\right)^{2 / 3}\right. \\
& \left.-P_{1}^{1 / 3} P_{2}^{2 / 3} P_{3}-5 \text { perm }\right] .
\end{aligned}
$$

In order to convert this to the matter bispectrum smoothed with the window function $W_{R}$, we use the Poisson equation (again, valid on subhorizon scales):

$$
\begin{aligned}
\delta_{R}(\mathbf{k}, z) & =\frac{2}{3} \frac{k^{2}}{(1+z) H_{0}^{2} \Omega_{m}} T(k) g_{*}(z) \tilde{W}_{R}(k) \Phi\left(\mathbf{k}, z_{*}\right) \\
& \equiv \mathcal{M}_{R}(k, z) \Phi\left(\mathbf{k}, z_{*}\right),
\end{aligned}
$$

where $T(k)$ is the matter transfer function, and $g_{*}(z) \propto$ $(1+z) D(z)$ is the potential growth factor normalized to unity at last scattering. Then, power spectra and bispectra are related by

$$
\begin{aligned}
& P_{R}(k, z)=\mathcal{M}_{R}^{2}(k, z) P_{\Phi}(k), \\
& B_{R}\left(k_{1}, k_{2}, k_{3} ; z\right)= \mathcal{M}_{R}\left(k_{1}, z\right) \mathcal{M}_{R}\left(k_{2}, z\right) \mathcal{M}_{R}\left(k_{3}, z\right) \\
& \times B_{\Phi}\left(k_{1}, k_{2}, k_{3}\right) .
\end{aligned}
$$

\section{APPENDIX B: DERIVATION OF THE VELOCITY DIFFERENCE DISTRIBUTION}

In this appendix we present some details on the derivation of the relative velocity distribution for peaks, starting from Eq. (30). Expressing the Dirac and Heaviside functions in terms of their Fourier transforms, using $\delta_{R}=$ $-\nabla \mathbf{u}_{R}$, and using Eq. (34) as well as partial integration, we can write the velocity probability distribution of peaks as

$$
\begin{aligned}
\mathcal{P}\left(\bar{u}_{r 1}, \bar{u}_{r 2} ; \mathbf{x}_{1}, \mathbf{x}_{2}\right)= & \int_{\nu \sigma_{R}} d \alpha_{1} \int_{\nu \sigma_{R}} d \alpha_{2}\left(\prod_{r=1}^{4} \int \frac{\mathrm{d} \phi_{r}}{2 \pi}\right) \\
& \times \exp \left(-i \alpha_{r} \phi^{r}\right) Z[\mathbf{J}],
\end{aligned}
$$




$$
\begin{gathered}
J^{i}(\mathbf{x})=\phi_{1} \partial^{i} W_{R}\left(\left|\mathbf{x}-\mathbf{x}_{1}\right|\right)+\phi_{2} \partial^{i} W_{R}\left(\left|\mathbf{x}-\mathbf{x}_{2}\right|\right), \\
+\phi_{3} W_{R}\left(\left|\mathbf{x}-\mathbf{x}_{1}\right|\right) \hat{r}^{i}+\phi_{4} W_{R}\left(\left|\mathbf{x}-\mathbf{x}_{2}\right|\right) \hat{r}^{i} .
\end{gathered}
$$

Here, we have defined $\alpha_{3} \equiv \bar{u}_{r 1}, \alpha_{4} \equiv \bar{u}_{r 2}$ in order to allow for more compact notation, and used the Einstein summing convention. In the following we adopt the notation of [13]. The current $\mathbf{J}$ is chosen to pick out the smoothed density field at $\mathbf{x}_{1}$ and $\mathbf{x}_{2}$ (via the $\phi_{1}, \phi_{2}$ terms), and the smoothed velocity field value at $\mathbf{x}_{1}$ and $\mathbf{x}_{2}$ (via the $\phi_{3}, \phi_{4}$ terms). One can then use Eq. (35) to convert this into an exponential of a sum over smoothed mixed density and velocity correlations. Extracting the terms proportional to $\phi_{r}^{2}, r=1 \ldots 4$, which correspond to the variance of the smoothed density and velocity fields, leaves the $\phi$ integrals as Fourier transforms of Gauss exponentials. We obtain

$$
\begin{aligned}
\mathcal{P}\left(\bar{u}_{r 1}, \bar{u}_{r 2} ; \mathbf{x}_{1}, \mathbf{x}_{2}\right)= & \frac{1}{(2 \pi)^{2} \sigma_{u}^{2}} \int_{\nu \sigma_{R}} d \alpha_{1} \int_{\nu \sigma_{R}} d \alpha_{2} \exp \left\{\frac{1}{2} \sum_{i \neq j}^{4} w_{R, i j}^{(2)} \frac{\partial^{2}}{\partial \alpha_{i} \partial \alpha_{j}}+\sum_{n=3}^{\infty} \frac{(-1)^{n}}{n !} \sum_{\left[i_{n}\right]}^{4} w_{R, i_{1} \ldots i_{n}}^{(n)} \frac{\partial^{n}}{\partial \alpha_{i_{1}} \ldots \partial \alpha_{i_{n}}}\right\} \\
& \times \exp \left(-\frac{1}{2} \alpha_{r} \alpha^{r}\right) .
\end{aligned}
$$

Here, we have denoted ordered sequences of integers $i_{1}, \ldots, i_{n}$, with $\left[i_{n}\right]$, and the sum runs over all those ordered sequences with each index running from 1 to 4 . Furthermore, we have defined

$$
\begin{gathered}
w_{R, i_{1} \ldots i_{n}}^{(n)} \equiv \xi_{R, i_{1} \ldots i_{n}}^{n} \sigma_{R, i_{1}}^{-1} \ldots \sigma_{R, i_{n}}^{-1} \\
\xi_{R, i_{1} \ldots i_{n}}^{(n)} \equiv\left(\prod_{j=1}^{n} \int \mathrm{d}^{3} \mathbf{x}_{j} W_{\left(i_{j}\right)}^{k_{j}}\left(\left|\mathbf{x}_{j}-\mathbf{r}_{i_{j}}\right|\right)\right) \xi_{k_{1} \ldots k_{n}}^{(n)}\left(\mathbf{x}_{1}, \ldots \mathbf{x}_{n}\right), \\
W_{(i)}^{k}(\mathbf{x}) \equiv \begin{cases}\partial^{k} W_{R}(\mathbf{x}), & i=1,2 \\
\hat{r}^{k} W_{R}(\mathbf{x}), & i=3,4\end{cases} \\
\sigma_{R, i} \equiv \begin{cases}\sigma_{R}, & i=1,2 \\
\sigma_{u}, & i=3,4\end{cases}
\end{gathered}
$$

This is a formidable array of definitions, which however allows us to keep the treatment general. In particular, the extension to higher-order velocity distributions, or mixed peak density and velocity distributions is straightforward. The reduced correlation functions $w_{R, i_{1} \ldots i_{n}}^{(n)}$ determine the strength of correlations between the different variables (density and velocity at $\mathbf{x}_{1}, \mathbf{x}_{2}$ ).

In evaluating the derivatives with respect to $\alpha_{j}$, it is useful to define the following function:

$$
\begin{aligned}
f_{m}(\nu) \equiv(-1)^{m} \int_{\nu \sigma_{R}} d \alpha\left(\frac{d}{d \alpha}\right)^{m} \exp \left(-\frac{1}{2} \alpha^{2}\right), & m \geq 1, \\
& =\frac{1}{\sqrt{2^{m-1}}} \mathrm{H}_{m-1}(\nu / \sqrt{2}) \exp \left(-\frac{1}{2} \nu^{2}\right),
\end{aligned}
$$

where $\mathrm{H}_{m}$ denotes the Hermite polynomials. We then define the following coefficients:

$$
a_{m} \equiv f_{m}(\nu) \approx \nu^{m-1} \exp \left(-\nu^{2} / 2\right), \quad m \geq 1,
$$

$$
\begin{gathered}
a_{0} \equiv \sqrt{\pi / 2} \operatorname{erfc}(\nu / \sqrt{2}) \approx \nu^{-1} \exp \left(-\nu^{2} / 2\right), \\
b_{m} \equiv f_{m+1}\left(\bar{u}_{r 1} / \sigma_{u}\right), \quad m \geq 0, \\
c_{m} \equiv f_{m+1}\left(\bar{u}_{r 2} / \sigma_{u}\right), \quad m \geq 0 .
\end{gathered}
$$

The $a_{m}(m \geq 1)$ coefficients are related to the bias parameters of the density peaks, as will be shown below. Note that they differ by factors of $(2 \pi)^{1 / 2}$ from those defined in [13]. The second approximate equality in Eqs. (B11) and (B12) is valid in the high-peak limit, $\nu \gg 1$, which we will assume throughout. Of course, we do not make this approximation for the velocities $\bar{u}_{r 1}, \bar{u}_{r 2}$. The first few velocity coefficients are

$$
\begin{gathered}
b_{0}=\exp \left(\bar{u}_{r 1}^{2} / 2 \sigma_{u}^{2}\right), \\
b_{1}=\frac{\bar{u}_{r 1}}{\sigma_{u}} \exp \left(\bar{u}_{r 1}^{2} / 2 \sigma_{u}^{2}\right), \\
b_{2}=\left(\frac{\bar{u}_{r 1}^{2}}{\sigma_{u}^{2}}-1\right) \exp \left(\bar{u}_{r 1}^{2} / 2 \sigma_{u}^{2}\right),
\end{gathered}
$$

and correspondingly for $c_{0}, c_{1}, c_{2}$.

For the peak correlation functions it is possible, by careful definition of different sets of integers, to expand the exponential in Eq. (B4), and reorder the terms into a sum over powers of $a_{i}$ [13]. In our case, this expression would become quite cumbersome however due to the different types of $\alpha$ parameters for peak density and velocity. Such an expression does not appear to be particularly useful. Instead, we make the assumption that the smoothed correlation functions $w_{R}^{(n)}$ are much less than unity, so that only the linear term of the expansion of the exponential needs to be kept. This will be appropriate, in particular, in our case since we are interested in large scales where the correlations are small. It is then straightforward to perform the $\alpha$ integrals. This assumption also means that the peak density field and its velocity distribution are close 
to Gaussian. Thus, we are perturbing the joint multivariate Gaussian distribution for the density and velocity fields, assuming that the non-Gaussianity is small; this will naturally lead us to an Edgeworth expansion of the velocity distribution. Algebraically, using our definitions above, each derivative $\partial^{n} / \partial \alpha_{i}^{n}$ (including $n=0$ ) in Eq. (B4) is replaced by $a_{n}$, if $i=1,2, b_{n}$ if $i=3$, and $c_{n}$ if $i=4$.

Analogous to (but much simpler than) the above derivation, one can obtain an expression for the peak density Eq. (28) in terms of the density $n$-point functions:

$$
\begin{aligned}
n_{\mathrm{pk}}(\nu)=(2 \pi)^{-1 / 2} \int_{\nu} d \alpha \exp \left\{\sum_{n=3}^{\infty} \frac{(-1)^{n}}{n !} w_{R}^{(n)}(0, \ldots, 0) \frac{\partial^{n}}{\partial \alpha^{n}}\right\} \\
\times e^{-\alpha^{2} / 2}, \\
\approx(2 \pi)^{-1 / 2}\left(a_{0}+\frac{1}{6} w_{R}^{(3)}(0,0,0) a_{3}\right) \\
\approx \frac{e^{-\nu^{2} / 2}}{\sqrt{2 \pi \nu^{2}}}\left(1+\frac{\nu^{3}}{6} \frac{\xi_{R}^{(3)}(0)}{\sigma_{R}^{3}}\right),
\end{aligned}
$$

where for the second equality we have again assumed small correlations and truncated the series at the threepoint function $\xi_{R}^{(3)}$. In the third equality, we have further used the $\nu \gg 1$ limit.

We can now assemble the velocity distribution $\mathcal{P}\left(\bar{u}_{r 1}, \bar{u}_{r 2} ; \mathbf{x}_{1}, \mathbf{x}_{2}\right)$, collecting all nonvanishing two-point and three-point terms. First, we order terms in the following way:

$$
\begin{aligned}
\mathcal{P}\left(\bar{u}_{r 1}, \bar{u}_{r 2} ; \mathbf{x}_{1}, \mathbf{x}_{2}\right)= & \left\{A+B \frac{\bar{u}_{r 2}-\bar{u}_{r 1}}{\sigma_{u}^{2}}+C \frac{\bar{u}_{r 1} \bar{u}_{r 2}}{\sigma_{u}}\right. \\
& +D\left[\frac{\bar{u}_{r 1}}{\sigma_{u}}\left(\frac{\bar{u}_{r 2}^{2}}{\sigma_{u}^{2}}-1\right)-\frac{\bar{u}_{r 2}}{\sigma_{u}}\left(\frac{\bar{u}_{r 1}^{2}}{\sigma_{u}^{2}}-1\right)\right] \\
& \left.+E\left(\frac{\bar{u}_{r 1}^{2}}{\sigma_{u}^{2}}-1\right)\right\} \frac{1}{2 \pi \sigma_{u}^{2}} \\
& \times \exp \left(-\frac{\bar{u}_{r 1}^{2}+\bar{u}_{r 2}^{2}}{2 \sigma_{u}^{2}}\right) .
\end{aligned}
$$

The coefficients of the different velocity terms are given by

$$
\begin{gathered}
A=1+b_{L}^{2} \xi_{R}^{\delta \delta}(r)+b_{L}^{3}\left[\frac{1}{3} \xi_{R}^{\delta \delta \delta}(0)+\xi_{R}^{\delta \delta \delta}\left(\mathbf{x}_{1}, \mathbf{x}_{1}, \mathbf{x}_{2}\right)\right] \\
-\frac{1}{3} b_{L}^{3} \xi_{R}^{\delta \delta \delta}(0), \\
B=\frac{b_{L}}{\sigma_{u}} \xi_{R}^{u \delta}(r)+\frac{b_{L}^{2}}{2 \sigma_{u}}\left[\xi_{R}^{\delta \delta u}\left(\mathbf{x}_{1}, \mathbf{x}_{1}, \mathbf{x}_{2}\right)\right. \\
\left.+2 \xi_{R}^{\delta \delta u}\left(\mathbf{x}_{1}, \mathbf{x}_{2}, \mathbf{x}_{2}\right)\right] \\
C=-\frac{\xi_{R}^{u u}(r)}{\sigma_{u}^{2}}+\frac{2 b_{L}}{\sigma_{u}^{2}} \xi_{R}^{\delta u u}\left(\mathbf{x}_{1}, \mathbf{x}_{1}, \mathbf{x}_{2}\right), \\
D=-\frac{1}{2 \sigma_{u}^{3}} \xi_{R}^{u u u}\left(\mathbf{x}_{1}, \mathbf{x}_{1}, \mathbf{x}_{2}\right),
\end{gathered}
$$

$$
E=\frac{b_{L}}{\sigma_{u}^{2}}\left[\xi_{R}^{\delta u u}(0)+\xi_{R}^{\delta u u}\left(\mathbf{x}_{1}, \mathbf{x}_{2}, \mathbf{x}_{2}\right)\right] .
$$

Here, we have defined the first order Lagrangian bias $b_{L} \equiv$ $\nu / \sigma_{R}$. The last term for $A$ comes from the normalization by the number of peaks, Eq. (B20). We can now evaluate Eq. (31) to obtain the peak correlation function (in real space),

$$
1+\xi_{\mathrm{pk}}(r)=b_{L}^{2} \xi_{R}^{\delta \delta}(r)+b_{L}^{3} \xi_{R}^{\delta \delta \delta}(r),
$$

recovering the well-known expression from, e.g., [6]. Correspondingly, the real-space peak power spectrum reads

$$
P_{\mathrm{pk}}(k)=b_{L}^{2} P_{R}(k)+b_{L}^{3} \mathcal{P}^{\delta \delta \delta}(k) .
$$

In order to obtain the matter-weighted velocities, we have to divide by $\left(1+\xi_{\mathrm{pk}}\right)$ [Eq. (32)]. This yields

$$
A^{\prime}=A-b_{L}^{2} \xi_{R}^{\delta \delta}(r)-b_{L}^{3} \xi_{R}^{\delta \delta \delta}\left(\mathbf{x}_{1}, \mathbf{x}_{1}, \mathbf{x}_{2}\right)=1 .
$$

In this way, $\mathcal{P}_{\delta u}$ is properly normalized to second order in the correlations. Performing the integral in Eq. (32) term by term, we obtain

$$
\begin{aligned}
\mathcal{P}_{\delta u}\left(\delta u ; \mathbf{x}_{1}, \mathbf{x}_{2}\right)= & \left\{1+B \frac{\delta u}{\sigma_{u}}+(E-C) \frac{1}{4}\left(\frac{\delta u^{2}}{\sigma_{u}^{2}}-2\right)\right. \\
& \left.+D \frac{\delta u}{4 \sigma_{u}}\left(\frac{\delta u^{2}}{\sigma_{u}^{2}}-6\right)\right\} \frac{1}{\sqrt{4 \pi \sigma_{u}^{2}}} \\
& \times \exp \left(-\frac{\delta u^{2}}{4 \sigma_{u}^{2}}\right) .
\end{aligned}
$$

Clearly, this expansion is equivalent to the Edgeworth expansion [Eq. (42)], and the coefficients $B$ through $E$ are easily related to the moments of $\delta u$. Using Eq. (16) again to express the various three-point correlations, we have

$$
\langle\delta u\rangle_{\mathrm{pk}}=2 B \sigma_{u}=2 b_{L} \xi_{R}^{u \delta}(r)+b_{L}^{2}\left[\xi_{R}^{\delta \delta u}(r)-2 \xi_{R}^{\delta u \delta}(r)\right],
$$

$$
\begin{aligned}
&\left\langle\delta u^{2}\right\rangle_{\mathrm{pk}}= 2(1+E-C) \sigma_{u}^{2} \\
&= 2\left[\sigma_{u}^{2}+\xi_{R}^{u u}(r)+b_{L} \xi_{R}^{u u} \delta(0)+b_{L} \xi_{R}^{u u \delta}(r)\right. \\
&\left.-2 b_{L} \xi_{R}^{\delta u u}(r)\right], \\
&\left\langle\delta u^{3}\right\rangle_{\mathrm{pk}}=12 D \sigma_{u}^{3}=6 \xi_{R}^{u u u}(r), \\
&\left\langle\delta u^{4}\right\rangle_{\mathrm{pk}}-3\left\langle\delta u^{2}\right\rangle_{\mathrm{pk}}^{2}=0 .
\end{aligned}
$$

These moments agree precisely with those derived in Sec. III A. 
[1] N. Kaiser, Mon. Not. R. Astron. Soc. 227, 1 (1987).

[2] K. B. Fisher, Astrophys. J. 448, 494 (1995).

[3] J.N. Fry and E. Gaztanaga, Astrophys. J. 413, 447 (1993).

[4] V. Desjacques and U. Seljak, Classical Quantum Gravity 27, 124011 (2010).

[5] N. Dalal, O. Doré, D. Huterer, and A. Shirokov, Phys. Rev. D 77, 123514 (2008).

[6] S. Matarrese and L. Verde, Astrophys. J. Lett. 677, L77 (2008).

[7] A. Slosar, C. Hirata, U. Seljak, S. Ho, and N. Padmanabhan, J. Cosmol. Astropart. Phys. 08 (2008) 031.

[8] P. Creminelli, A. Nicolis, L. Senatore, M. Tegmark, and M. Zaldarriaga, J. Cosmol. Astropart. Phys. 05 (2006) 004.

[9] P.D. Meerburg, J.P. van der Schaar, and P. Stefano Corasaniti, J. Cosmol. Astropart. Phys. 05 (2009) 018.

[10] P. Catelan and R. J. Scherrer, Astrophys. J. 445, 1 (1995).

[11] V. Desjacques, Phys. Rev. D 78, 103503 (2008).

[12] V. Desjacques and R. K. Sheth, Phys. Rev. D 81, 023526 (2010).

[13] S. Matarrese, F. Lucchin, and S. A. Bonometto, Astrophys. J. Lett. 310, L21 (1986).

[14] B. Grinstein and M. B. Wise, Astrophys. J. 310, 19 (1986).

[15] T. Matsubara, Astrophys. J. 584, 1 (2003).
[16] L. Verde and S. Matarrese, Astrophys. J. Lett. 706, L91 (2009).

[17] R. J. Scherrer and E. Bertschinger, Astrophys. J. 381, 349 (1991).

[18] H. A. Feldman, R. Watkins, and M. J. Hudson, Mon. Not. R. Astron. Soc. (to be published).

[19] A. Kashlinsky, F. Atrio-Barandela, H. Ebeling, A. Edge, and D. Kocevski, Astrophys. J. Lett. 712, L81 (2010).

[20] J. Lee and E. Komatsu, Astrophys. J. 718, 60 (2010).

[21] R. Scoccimarro, Phys. Rev. D 70, 083007 (2004).

[22] T. Y. Lam, V. Desjacques, and R. K. Sheth, Mon. Not. R. Astron. Soc. 402, 2397 (2010).

[23] R. E. Smith, J. A. Peacock, A. Jenkins, S. D. M. White, C. S. Frenk, F. R. Pearce, P. A. Thomas, G. Efstathiou, and H. M. P. Couchman, Mon. Not. R. Astron. Soc. 341, 1311 (2003).

[24] P. McDonald and U. Seljak, J. Cosmol. Astropart. Phys. 10 (2009) 007.

[25] M. White, Y. Song, and W. J. Percival, Mon. Not. R. Astron. Soc. 397, 1348 (2009).

[26] P. Zhang, M. Liguori, R. Bean, and S. Dodelson, Phys. Rev. Lett. 99, 141302 (2007).

[27] Y. Song and W. J. Percival, J. Cosmol. Astropart. Phys. 10 (2009) 004. 